\title{
A Comparative Performance Analysis of Two Printed Circular Arrays for Power-Based Vehicle Localization Applications
}

\author{
Mohammad S. Sharawi, ${ }^{1}$ Farooq Sultan, ${ }^{1}$ and Daniel N. Aloi ${ }^{2}$ \\ ${ }^{1}$ Electrical Engineering Department, King Fahd University of Petroleum and Minerals, Dhahran 31261, Saudi Arabia \\ ${ }^{2}$ Electrical and Computer Engineering Department, Oakland University, Rochester, MI 48309, USA
}

Correspondence should be addressed to Mohammad S. Sharawi, msharawi@kfupm.edu.sa

Received 2 April 2012; Revised 1 August 2012; Accepted 24 August 2012

Academic Editor: Deb Chatterjee

Copyright (C) 2012 Mohammad S. Sharawi et al. This is an open access article distributed under the Creative Commons Attribution License, which permits unrestricted use, distribution, and reproduction in any medium, provided the original work is properly cited.

\begin{abstract}
A comparative study of the performance characteristics of a printed 8-element V-shaped circular antenna array and an 8-element Yagi circular array operating at $2.45 \mathrm{GHz}$ for vehicular direction finding applications is presented. Two operating modes are investigated; switched and phased modes. The arrays were fabricated on FR-4 substrates with $0.8 \mathrm{~mm}$ thickness. Measured and simulated results were compared. Radiation gain patterns were measured on a $1 \mathrm{~m}$ diameter ground plane that resembles the rooftop of a vehicle. The HPBW of the Yagi was found to be about $3^{\circ}$ narrower than its $\mathrm{V}$-shaped counterpart when measured above a reflecting ground plane and operated in switched mode. The printed V-shaped antenna array offers $2.5 \mathrm{~dB}$ extra gain compared to the printed Yagi array.
\end{abstract}

\section{Introduction}

Increased usage of tracking devices in cars raised the need for the development of highly accurate position determination systems. One of the basic requirements for radio frequency (RF) vehicular localization is the accurate estimation of the direction of arrival (DOA) of the incoming RF signal [1]. Recent advances in smart antenna technologies-antennas with digital signal processing (DSP) equipment-enabled the enhancement of antenna coverage and efficient utilization of the transmitted power. In a bid to accommodate the maximum possible number of users, cellular service providers are utilizing smart antenna systems to shape the radiation patterns to focus their transmit power in a given region.

Traditionally, highly accurate DOA systems require the use of multiple receivers with separate antennas integrated with DSP algorithms like the multiple signal classification (MUSIC) and rotational invariance techniques (ESPRIT) $[2,3]$. The use of multiple receivers dictated the size of the overall system to be large making it difficult to deploy for several applications. For increased practicality, microstrip antenna arrays are used nowadays to save space and to decrease the overall profile of the system. As the number of cars globally increases beyond the 1 billion mark [4], parking problems in densely populated cities have started to increase. Indoor, multistory parking lots are being considered as one of the most efficient ways of providing parking space for the available cars without interrupting the traffic flow. An emerging application of DF systems is for car localization in huge parking lots. The majority of modern cars are equipped with remote entry systems which can help in locating the car when the transmitter (key) is triggered within a specific distance from the receiver (car). Global positioning system (GPS-) based DF systems have proved their usefulness in open air parking lots [5] but in multistory covered parking plazas GPS-based solutions become ineffective.

All of the above mentioned systems rely on a buzzer to guide the user to the car location. The buzzer might not be heard, not to mention that this solution will never work for hearing impaired people. An alternative approach is a system that determines the actual location of the car and sends a signal to the user that will show on a key fob (controller with lights and buttons) with his/her key chain pointing him/her to the correct direction of the location of the car. Reference [6] have proposed the design of a DF system based on a four 


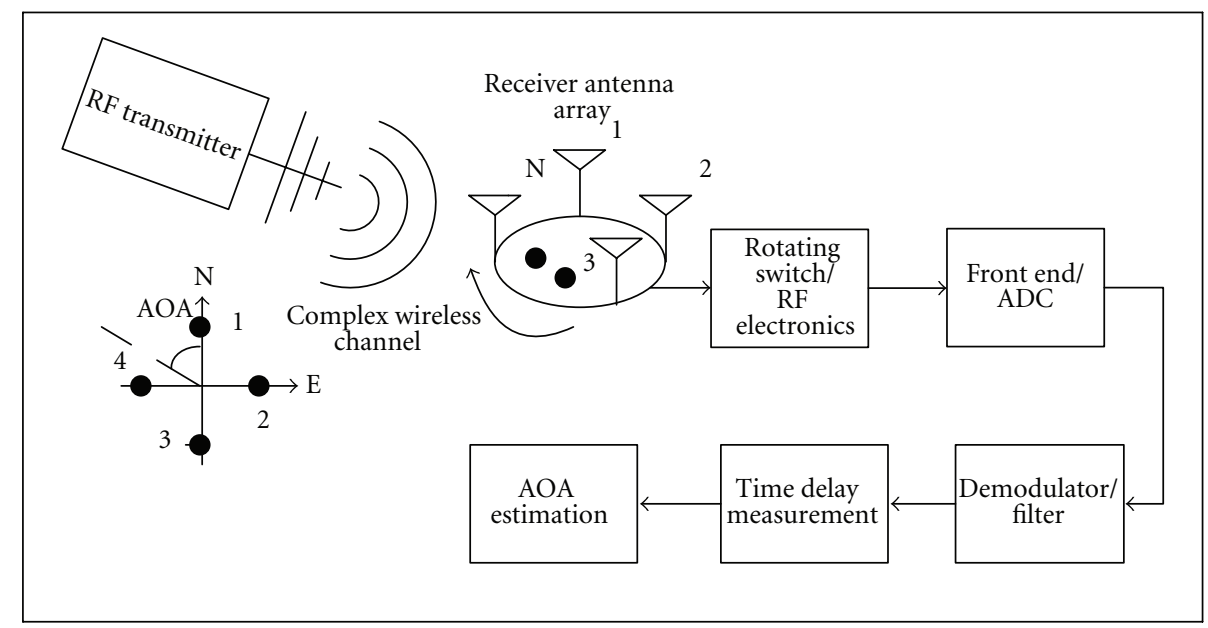

Figure 1: Block diagram of a single channel car localization system.

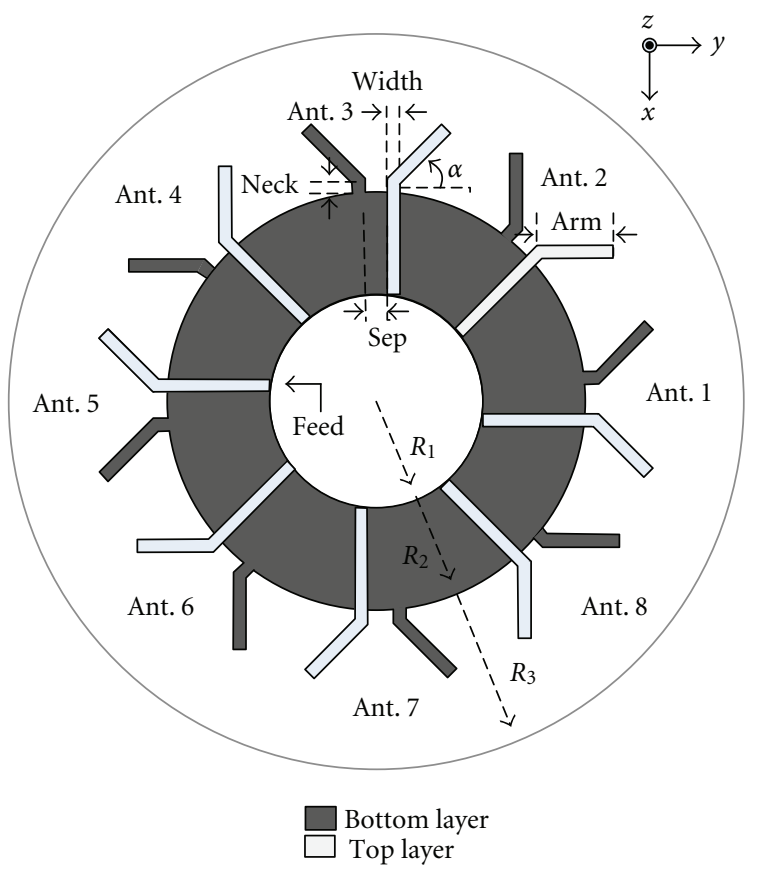

(a)

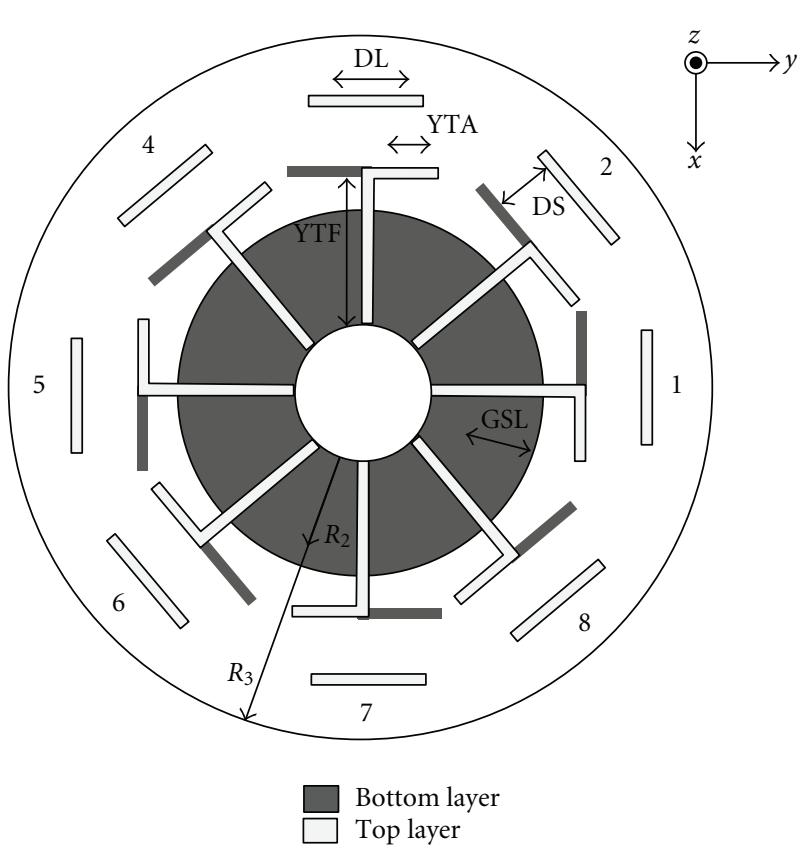

(b)

Figure 2: Designs for the printed arrays (a) 8-element V-shaped circular array and (b) 8-element Yagi circular array.

element linear array antenna with phase shifters for beam scanning. The beam scanning ability of this system is highly limited by the linear nature of the array and thus cannot scan more than $180^{\circ}$ in the azimuth plane. Considering a large-covered parking lot where the position of the car is not known, accurate localization requires the onboard DF system to be able to scan $360^{\circ}$ so that signals from the transmitter are received regardless of its position with respect to the receiver. In [7], the performance of an 8-element circular Yagi antenna array with two slightly different ground (GND) plane designs was proposed and analyzed. Since the application of this antenna requires hoisting it on top of a car, the roof of the car acts as a large ground plane and has a significant effect on the radiation pattern of the antenna. The authors did not take the effect of the ground plane into consideration. Moreover, the half power beamwidth (HPBW) offered by the circular Yagi array was much wider than needed for accurate localization. The block diagram of a single channel car localization system similar to that in [8] is shown in Figure 1. The work presented in this paper focuses solely on the antenna array section.

V-shaped wire antennas are known for their directive patterns when operated in pairs [9]. The design and characterization of printed V-shaped antenna elements is not found in literature. In this work we present the design, fabrication, and characterization of a V-shaped printed antenna array 
TABLE 1: Simulated and measured center frequency, $-10 \mathrm{~dB}$ matching bandwidth, and $\left|S_{11}\right|$ for the Yagi and the V-shaped antennas.

\begin{tabular}{|c|c|c|c|c|c|c|c|}
\hline \multirow{2}{*}{ Antenna type } & \multirow{2}{*}{ Antenna number } & \multicolumn{2}{|c|}{ Center frequency $(\mathrm{GHz})$} & \multicolumn{2}{|c|}{-10 dB Bandwidth (MHz) } & \multicolumn{2}{|c|}{$\left|S_{11}\right|(\mathrm{dB})$} \\
\hline & & Sim & Meas & Sim & Meas & Sim & Meas \\
\hline \multirow{8}{*}{ Yagi } & 1 & 2.347 & 2.41 & 365 & 297 & -21.4 & -35.8 \\
\hline & 2 & 2.397 & 2.41 & 369 & 290 & -22.6 & -28.7 \\
\hline & 3 & 2.397 & 2.41 & 365 & 310 & -22.1 & -32 \\
\hline & 4 & 2.397 & 2.41 & 362 & 296 & -21.6 & -24.2 \\
\hline & 5 & 2.399 & 2.40 & 365 & 334 & -21.6 & -36.7 \\
\hline & 6 & 2.397 & 2.41 & 360 & 292 & -22.5 & -26.3 \\
\hline & 7 & 2.393 & 2.41 & 358 & 298 & -21.6 & -34.5 \\
\hline & 8 & 2.399 & 2.41 & 369 & 310 & -22.9 & -32.2 \\
\hline \multirow{8}{*}{ V-Shaped } & 1 & 2.5 & 2.51 & 304 & 240 & -17.3 & -25.7 \\
\hline & 2 & 2.5 & 2.51 & 300 & 238 & -17.0 & -25.3 \\
\hline & 3 & 2.5 & 2.51 & 300 & 240 & -17.1 & -25.6 \\
\hline & 4 & 2.5 & 2.50 & 301 & 235 & -16.9 & -26.3 \\
\hline & 5 & 2.5 & 2.51 & 300 & 233 & -17.1 & -26.2 \\
\hline & 6 & 2.5 & 2.51 & 302 & 240 & -17.7 & -25.6 \\
\hline & 7 & 2.5 & 2.50 & 301 & 235 & -17.2 & -25.2 \\
\hline & 8 & 2.5 & 2.51 & 301 & 238 & -17.8 & -25.8 \\
\hline
\end{tabular}

operating at $2.45 \mathrm{GHz}$. In addition this work provides a comparison between the performance of a printed 8-element circular Yagi antenna array and the proposed 8-element circular V-shaped antenna array for single channel vehicular direction finding applications. Simulation results for the HPBW, $-10 \mathrm{~dB}$ matching bandwidth, and the effect of the ground plane are presented and a comparison between the two array types is made. In addition, the antennas were fabricated and the gain patterns were measured at an outdoor antenna range facility at Oakland University, Michigan, USA. The arrays were excited under switched as well as phased modes of operation to allow for proper use in power based RF-DF applications.

The rest of the paper is organized as follows: Section 2 presents the modeling and designs of the two antenna arrays, Section 3 shows the comparative study of the simulated and the measured quantities for the two antennas without the presence of the ground plane and when a ground plane is inserted beneath the antenna arrays, and finally the paper concludes with Section 4.

\section{Antenna Array Designs}

The geometries of the printed V-shaped and the Yagi circular antenna arrays are shown in Figure 2. Both arrays were designed to operate in the $2.45 \mathrm{GHz}$ band.

The printed V-shaped circular antenna array (Figure 2(a)) was designed on an FR-4 substrate with $\epsilon_{r}=3.8$ and $0.8 \mathrm{~mm}$ thickness. The dimensions of the $\mathrm{V}$-shaped antenna array were (in $\mathrm{mm}$ ) $R 1=25, R 2=45$, $R 3=100$, Arm $=20$, neck $=3$, Width $=1.5$, Sep $=7$, and $\alpha=30^{\circ}$. A parametric study via simulations was conducted to investigate the effect of the printed V-shaped antenna parameters on its resonance and operating bandwidth. An increase in $\alpha$ as well as neck increases both the center frequency as well as the $-10 \mathrm{~dB}$ matching bandwidth. An increase in arm on the other hand, decreases the resonance frequency and decreases the offered bandwidth as well. Increasing sep decreases the resonant frequency while it increases the offered bandwidth. These parameters were tuned using HFSS. The dark elements and the circular ground plane are on the bottom side of the PCB while the white/light colored arms are on the top side.

For the printed Yagi antenna array, an FR-4 material with $\epsilon_{r}=4.0$ and a thickness of $0.8 \mathrm{~mm}$ was used; the dimensions of the design in $\mathrm{mm}$ (Figure $2(\mathrm{~b})$ ) were $R 1=25, R 2=51$, $R 3=100, \mathrm{YTF}=43, \mathrm{YTA}=26, \mathrm{DL}=32, \mathrm{DS}=10$, and $\mathrm{GSL}=19$. These values were optimized using a 3D full wave field simulator (HFSS). The feeder length (YTA) affects the resonance frequency as well as the operating bandwidth. If its length is increased both the resonance frequency and operating BW decreases. The distance between the director and the feeder affects the input matching of the feeder, and should be kept at least $10 \mathrm{~mm}$ away from the feeder. Increasing the director length decreases the HPBW while increasing the back lobe levels. The dark elements and the circular ground plane are on the bottom side of the PCB while the white/light colored arms are on the top side. Note that the antenna elements have been numbered arbitrarily from 1 to 8 and the same naming scheme is used in the following sections.

\section{Results and Discussions}

A snapshot of the two-fabricated antennas is presented in Figure 3. An Agilent HP8510C network analyzer was used to carry out the measurements of the two arrays in the microwave laboratory in the EE Department at KFUPM. Each of the eight ports of the array were excited individually while all other ports were terminated with $50 \Omega$. 


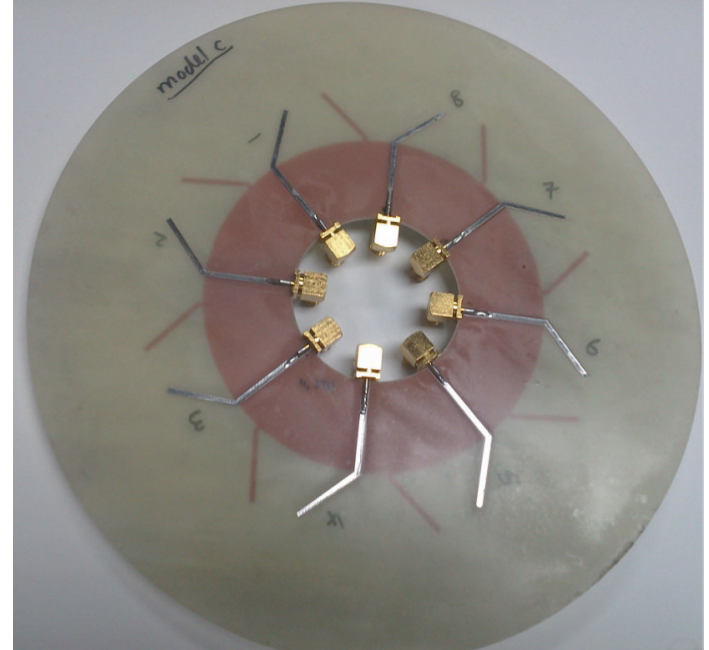

(a)

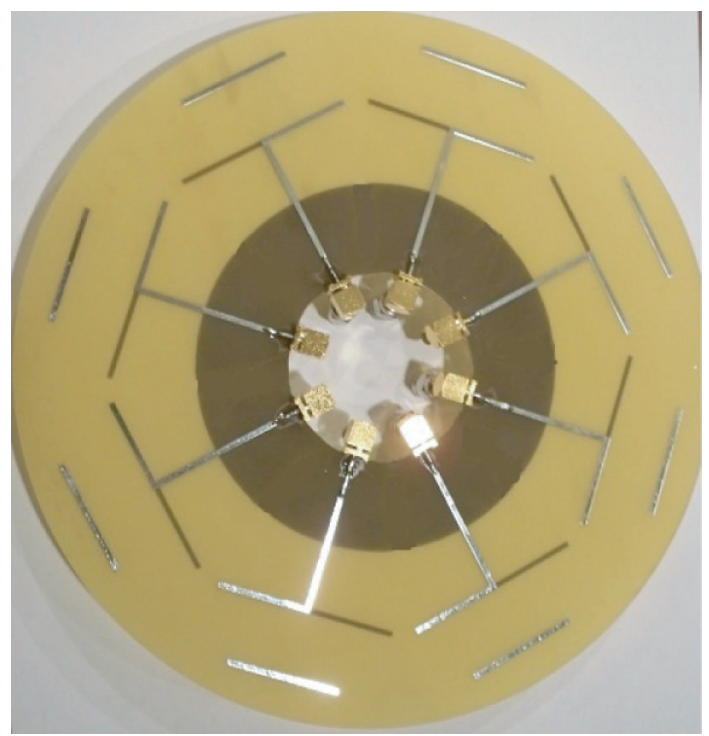

(b)

FIGURE 3: Fabricated printed antenna arrays: (a) 8-element Vshaped circular array and (b) 8-element Yagi circular array.

Table 1 presents the measured (meas) and simulated (sim) values for the resonance frequencies, HPBW, and $\left|S_{11}\right|$. Figure 4 shows the measured and simulated values of the reflection coefficients for elements 1 and 2 for both the Vshaped and the Yagi arrays. Good agreement is observed from the results.

Comparing the simulated and the measured results in Table 1, good agreement is observed. The $-10 \mathrm{~dB}$ matching BW of the measured antenna is lower than the corresponding simulated one. One of the possible reasons for this deviation can be the effect of the fabrication process and the presence of SMA connectors used to feed the antenna elements. Although great attention was paid while soldering the SMA connectors to the antenna arms, one can never achieve absolute accuracy while soldering by hand; this might also be a possible reason for the difference between the measured

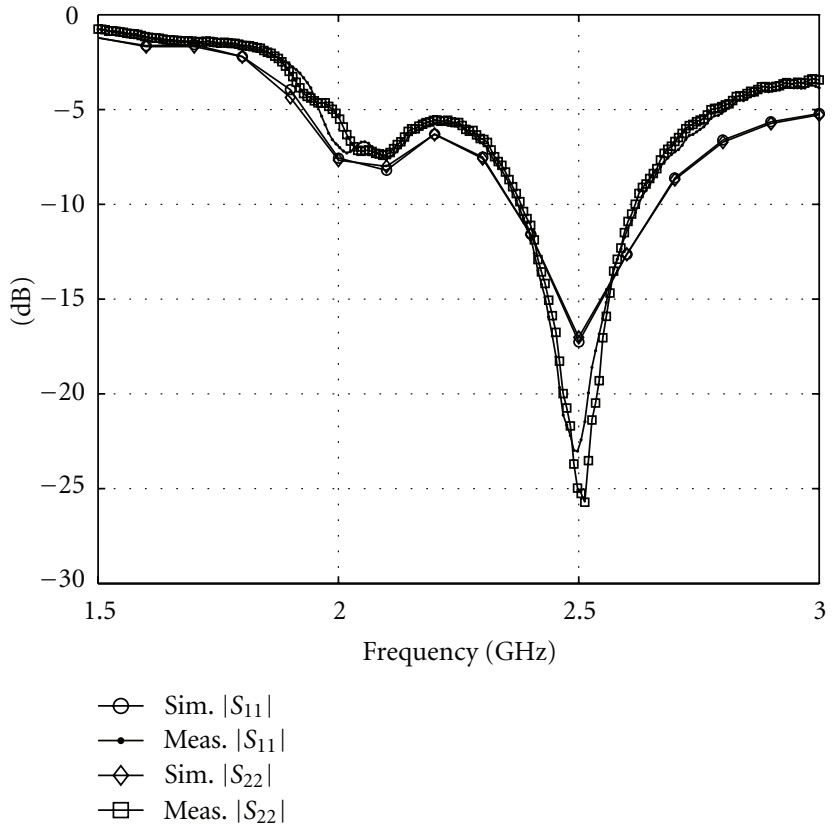

(a)

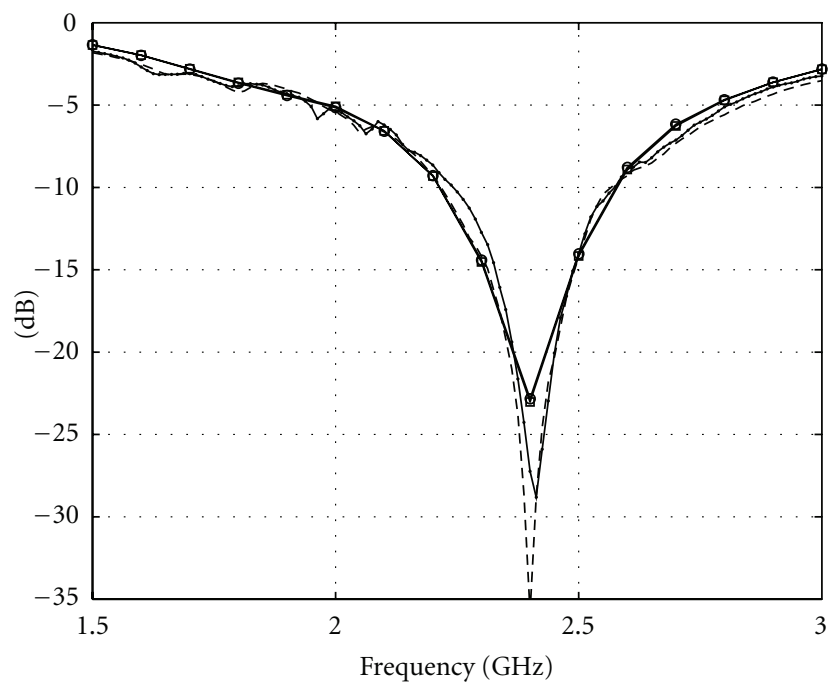

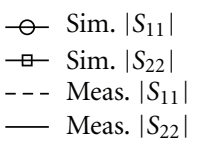

(b)

FIGURE 4: Simulated and measured reflection coefficient for elements 1 and 2 for (a) V-shaped antenna array and (b) Yagi antenna array.

and the simulated values. The difference between the $\left|S_{11}\right|$ values is believed to be attributed to the difference between the simulated and fabricated feed excitation mechanisms, the former was a feeding strip like a probe feed while the latter was a regular SMA connector.

3.1. Switched and Phased Modes of Operation without a Reflecting Ground Plane. In order to measure the radiation 


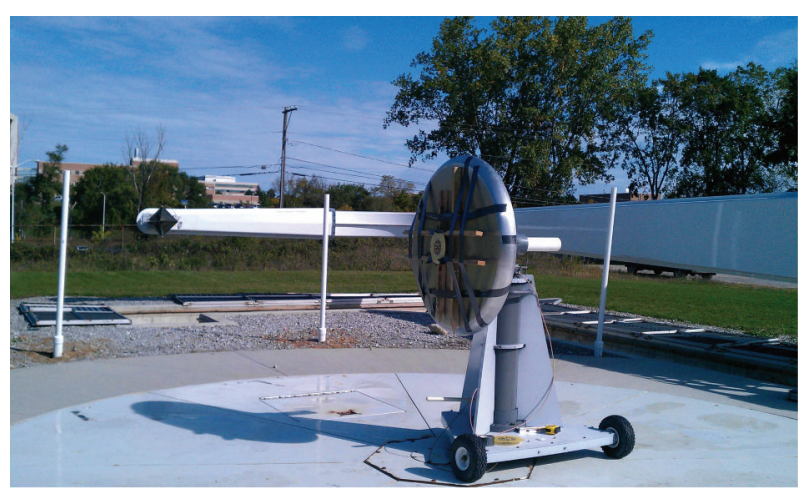

Figure 5: Antenna range at Oakland university.

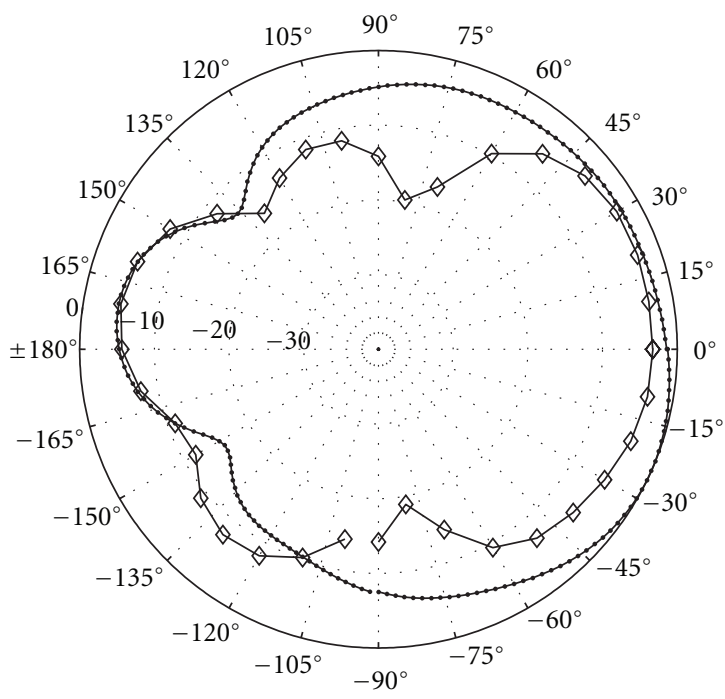

Figure 6: Normalized simulated directivity pattern comparison for the antennas without ground plane-dotted markers for V-shaped and diamond markers for Yagi pattern.

characteristics for the switched mode of operation of the Vshaped and the Yagi circular antenna arrays, the fabricated antennas were measured at an outdoor antenna range at Oakland university, Michigan, USA. Figure 5 shows the measurement set-up.

Since there are eight array elements spanning an azimuthal plane of $360^{\circ}$, it is designed such that each element provides directive radiation pattern along its azimuthal direction $(\phi)$ covering a sector of $45^{\circ}$. The antenna array is to be mounted on the car rooftop, the main region of interest in our work is the azimuth $(\phi)$ plane. All the measurements presented in the following Sections consider the azimuth plane patterns. For accurate localization in a switched mode operation of the array where only one element is excited at a time, it is required that the HPBW of each of the antenna elements of the array be as narrow as possible so that a clear distinction between signals received at adjacent antenna elements can be made in a power based RF-DF system. The switched mode of operation does not require complex electronics, only an RF switch/MUX along with a

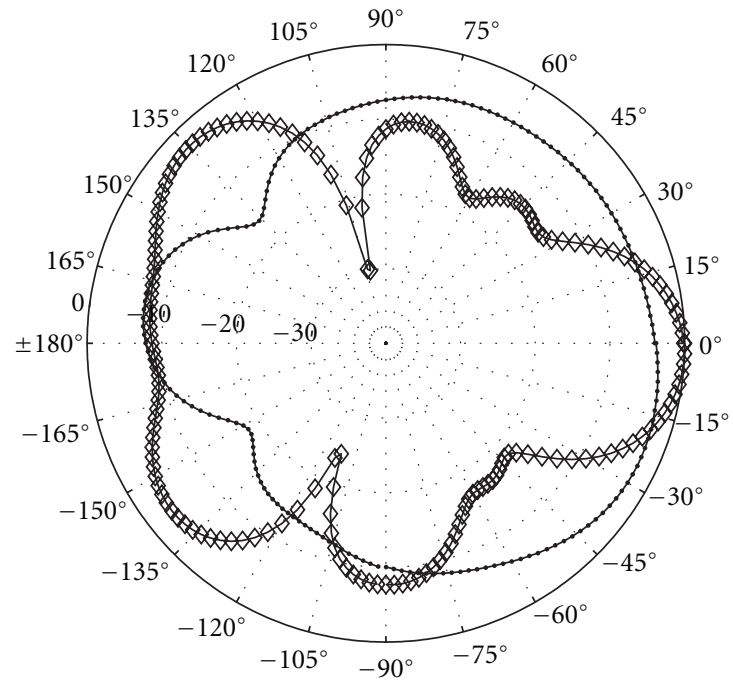

FIGURE 7: Normalized simulated directivity patterns for the Vshaped antenna array without ground plane-dotted markers for switched mode and diamond markers for phased mode.

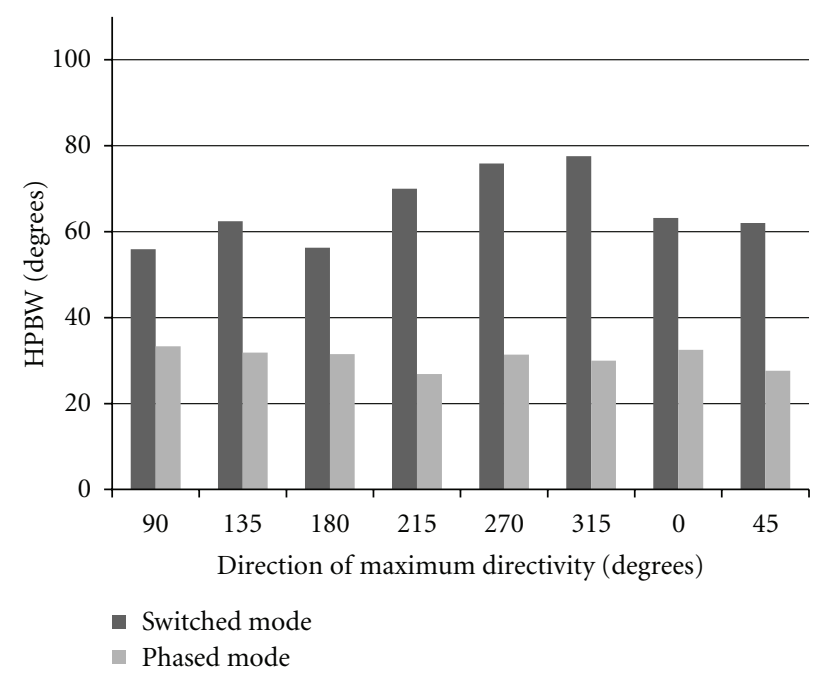

FIGURE 8: HPBW simulations comparison for the V-shaped antenna array placed over a ground plane.

power measurement IC (received signal strength indicatorRSSI), and a microcontroller are needed. In the comparison between the normalized azimuthal directivity patterns of the Yagi and the V-shaped antenna presented in Figure 6, we observe that the V-shaped antenna array provides higher HPBW when operated under switched mode. The HPBW for the Yagi was $100^{\circ}$ whereas that for the V-shaped array was found to be $120^{\circ}$. Antenna 7 (towards $\phi=0^{\circ}$ ) was excited for both arrays. The gain of the V-shaped array was approximately $2.5 \mathrm{dBs}$ higher than the Yagi array.

Unlike the switched mode of operation, phased mode requires exciting all the antenna elements simultaneously with equal excitation coefficients but varying phases. Analytical expressions to calculate the relative phases required 


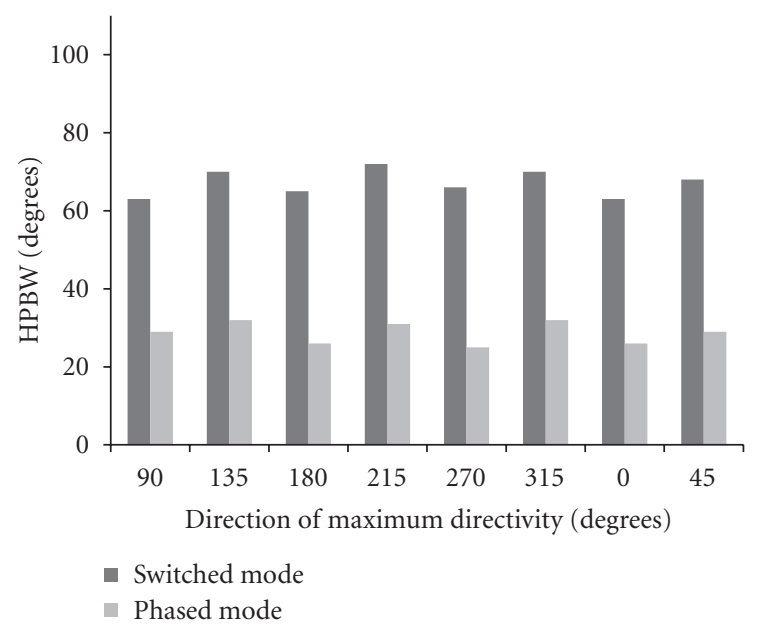

FIGURE 9: HPBW simulation comparison for the Yagi array placed $5 \mathrm{~cm}$ over the ground plane.

to achieve maximum gain/directivity in a given direction are found in [9]. Figure 7 shows the simulated azimuthal patterns $\mathrm{V}$-shaped antenna when operated under switched and phased modes of excitations; the HPBW for the switched mode was found to be approximately $100^{\circ}$ and for the phased mode was $34^{\circ}$. In addition, gain improvement of about $3 \mathrm{~dB}$ was observed. This improvement came at the cost of higher hardware complexity.

\subsection{Switched and Phased Modes of Operation with a Reflecting} Ground Plane. As mentioned in Section 1, the application of our antenna designs require that they are installed on the car roof. The roof, being made of metal, tends to reflect the energy radiated by the antenna array in the downward direction hence increasing the power radiated upwards and affecting the azimuthal patterns. In order to correctly model the effect of the car's roof on the performance of the antenna array, we have simulated the array models by placing a large conducting plane $(1 \mathrm{~m}$ in diameter) at a height of $5 \mathrm{~cm}$ below the array. These simulations mimic the behavior of the car roof on our antenna's performance and provide a much closer insight to their behavior. In addition to the simulations, the antenna arrays were measured by placing them over a $1 \mathrm{~m}$ diameter rolled edge ground plane at a height of $5 \mathrm{~cm}$ to measure the actual radiation patterns.

Figures 8 and 9 present a comparison between the simulated HPBW for the two excitation modes of the V-shaped array and the Yagi antenna array, respectively. For both the antenna arrays, a sharp decrease in the HPBW is observed when operated under the phased mode of excitation. The presence of ground plane reduced the HPBW by approximately $45^{\circ}$ on average for the V-shaped array (Figure 8), and approximately $35^{\circ}$ for the Yagi array (Figure 9) when operated in switched mode. In addition, the maximum gain for both arrays was reduced by a couple of $\mathrm{dBs}$ when operated in this mode. The HPBW for the phased mode of operation was not affected as much by the presence

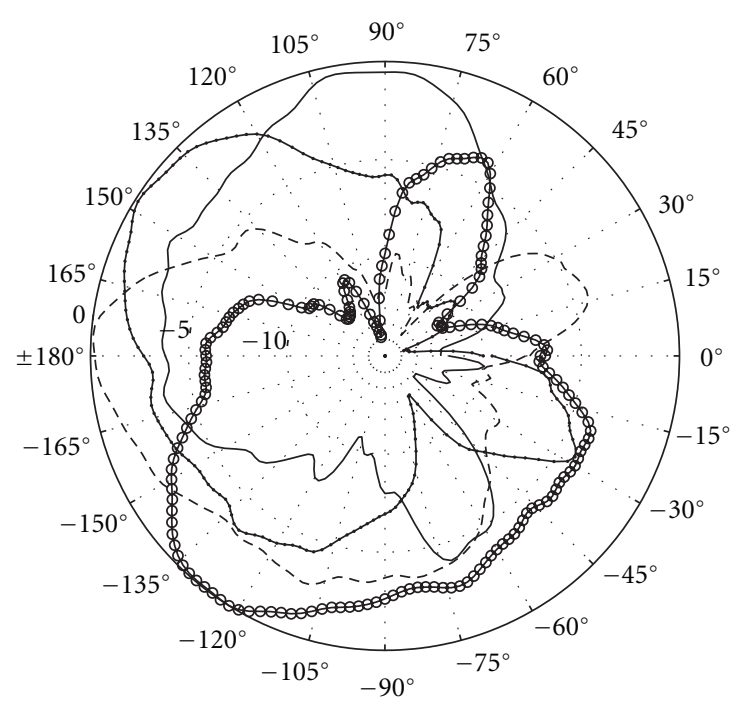

(a)

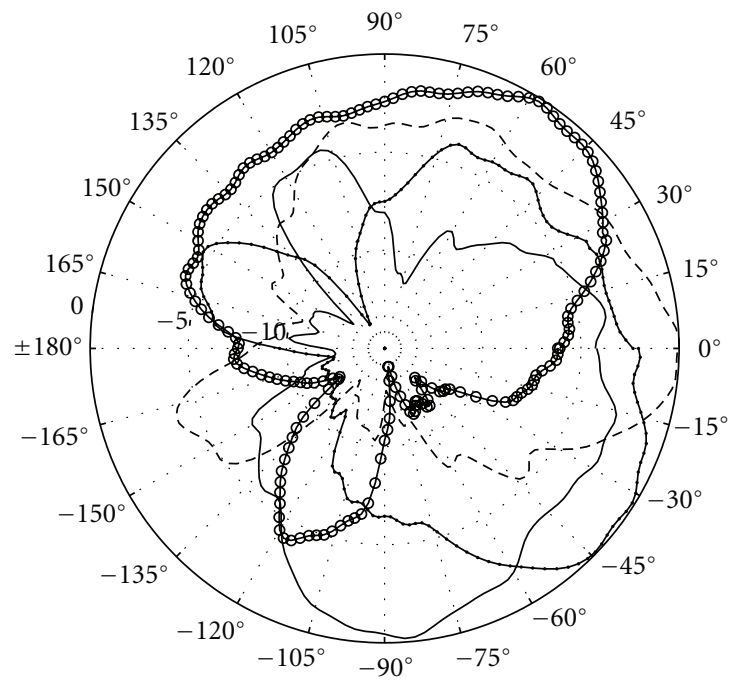

(b)

Figure 10: Simulated switched mode beams overlayed for the vshaped antenna (a) elements 1 to 4 and (b) elements 5 to 8. Solid (elements 1 and 5), dots (elements 2 and 6), dashes (elements 3 and 7), and circles (elements 4 and 8).

of the ground plane. This was expected albeit at the expense of higher cost electronics.

The switched mode beams for multiple elements of the V-shaped array with a GND plane $(5 \mathrm{~cm}$ below) are shown in Figure 10. Figure 10(a) shows the beams of the first 4 elements, while Figure 10(b) shows the beams of the remaining 4-elements. Please observe that some overlap will occur due to the larger than $45^{\circ} \mathrm{HPBW}$. But with proper electronics, the correct sector can be correctly chosen using the simple and cheap switch mode operation of the array (a quadrant rather a narrow sector). Figure 11 shows the simulated gain values as a function of frequency for the Yagi and V-shaped arrays. The V-shaped array offers 


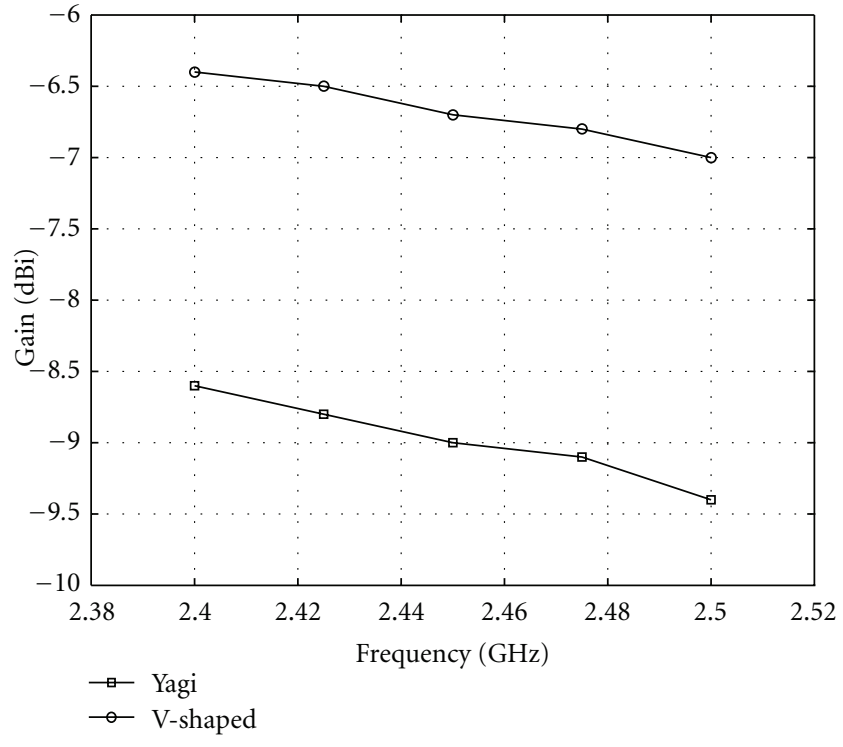

FIGURE 11: Simulated Gain values versus frequency for the V-shaped and Yagi arrays $5 \mathrm{~cm}$ over a $1 \mathrm{~m}$ diameter GND plane.

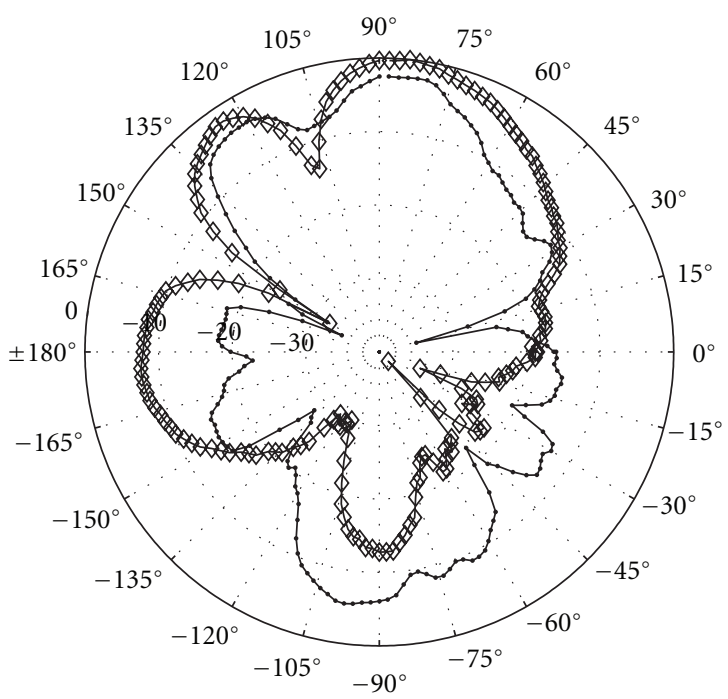

FIGURE 12: Normalized measured gain patterns for switched mode operation with ground plane-dotted markers for Yagi and diamond markers for V-shaped.

approximately $2.5 \mathrm{~dB}$ higher gain than the Yagi array. Please note that the gain values are low because the main beam is directed upwards due to the presence of the GND plane beneath the array. Gain values were taken at the elevation angle of $90^{\circ}$ representing the roof of a vehicle.

The normalized-measured gain patterns for the switched mode of operation and when element 1 is excited are shown in Figure 12 for both the Yagi and the V-shaped arrays when placed $5 \mathrm{~cm}$ above a $1 \mathrm{~m}$ diameter ground plane. While the Yagi elements provided better HPBW, the V-shaped elements provided higher gain values. In Addition the back lobe gain

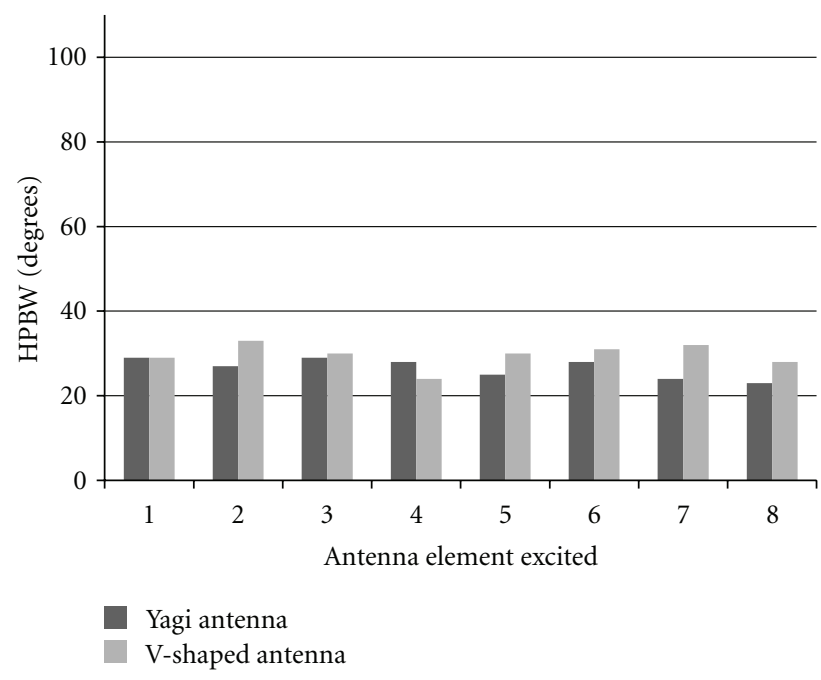

Figure 13: Measured HPBW comparison for the Yagi and the Vshaped array placed $5 \mathrm{~cm}$ over a ground plane operated under switched mode.

of the Yagi array was $5 \mathrm{~dB}$ higher than the backlobe gain of the V-shaped array.

The presence of the ground plane showed a sharp decrease in the HPBW of the antennas as compared to the case without a ground reflector for both arrays in the switched mode of operation. Figure 13 presents the measured HPBW comparison between the Yagi and the Vshaped antenna array operated under the switched mode and in the presence of the ground plane. It is clear that the Yagi array provides a narrower HPBW by an average of $3^{\circ}$ than the $\mathrm{V}$-shaped array. The measurements show that the HPBW are $50 \%$ less than their simulated counterparts; this is due to the presence of a sharp dip in the main lobe (Figure 12) that might be attributed to the mounting setup of the antennas. The mounting set-up consisted of duct tape as well as wooden bars to raise the antenna array above the GND plane and hold it in its place. The duct tape (considered as a new dielectric medium) might have affected the radiation characteristics and might have contributed to the dip observed. What is important here is that the presence of a GND plane below the arrays had resulted in narrower HPBW (since the maximum of the pattern was pushed upwards) that allows them to be used in powerbased-direction finding applications at lower cost.

\section{Conclusion}

A comparative performance analysis of two printed circular antenna arrays of V-shaped and Yagi elements has been presented in this paper. Both arrays were designed to operate at $2.45 \mathrm{GHz}$. Switched and phased modes of excitation were simulated for both antenna designs. The arrays are intended for use in power-based RF DF applications. Measurements under the switched mode reveal that the HPBW is lowered by the presence of a reflecting ground plane, this is of great 
importance for enhancing the sectorial resolution for such an application.

\section{Acknowledgment}

The authors would like to acknowledge the support provided by the Deanship of Scientific Research (DSR) at King Fahd University of Petroleum and Minerals (KFUPM), Dhahran, Saudi Arabia, for funding this work through Project no. SB101010.

\section{References}

[1] M. S. Sharawi and D. N. Aloi, "Characterizing the performance of single-channel Pseudo-Doppler direction finding systems at $915 \mathrm{MHz}$ for vehicle localization," International Journal of Communication Systems, vol. 24, no. 1, pp. 27-39, 2011.

[2] R. O. Schmidt, "Multiple emitter location and signal parameter estimation," IEEE Transactions on Antennas and Propagation, vol. 34, no. 3, pp. 276-280, 1986.

[3] R. Roy and T. Kailath, "ESPRIT-estimation of signal parameters via rotational invariance techniques," IEEE Transactions on Acoustics, Speech, and Signal Processing, vol. 37, no. 7, pp. 984995, 1989.

[4] D. Tencer, "Number of cars worldwide surpasses 1 billion: can the world handle this many wheels?" The Huffington Post Canada, September 2012.

[5] R. Christ, "Application and performance of personnel tracking systems," in Proceedings of the 43rd IEEE International Carnahan Conference on Security Technology, pp. 120-128, 2009.

[6] V. Rabinovich, "Direction finding system for automotive applications using small phased antenna array," Microwave and Optical Technology Letters, vol. 53, no. 10, pp. 2441-2446, 2011.

[7] M. S. Sharawi and D. N. Aloi, "Design of an 8-element switched mode circular antenna array for vehicular direction finding," in Proceedings of the IEEE 12th Annual Wireless and Microwave Technology Conference (WAMICON '11), pp. 1-4, Clearwater, Fla, USA, April 2011.

[8] D. N. Aloi and M. S. Sharawi, "Comparative analysis of singlechannel direction finding algorithms for automotive applications at $2400 \mathrm{MHz}$ in a complex reflecting environment," Physical Communication, vol. 3, no. 1, pp. 19-27, 2010.

[9] C. A. Balanis, Antenna Theory: Analysis and Design, John Wiley \& Sons, 3rd edition, 2005. 

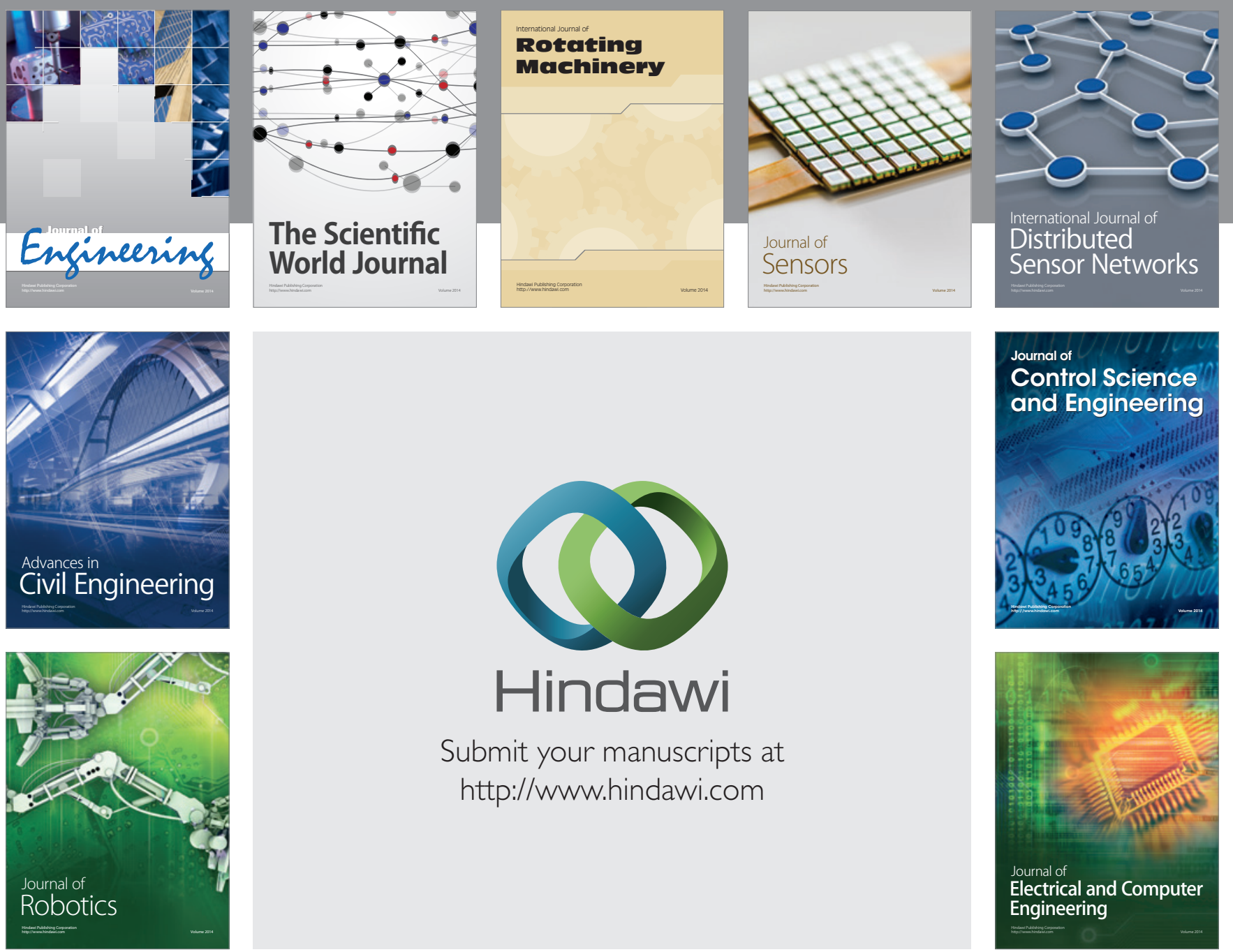

Submit your manuscripts at

http://www.hindawi.com
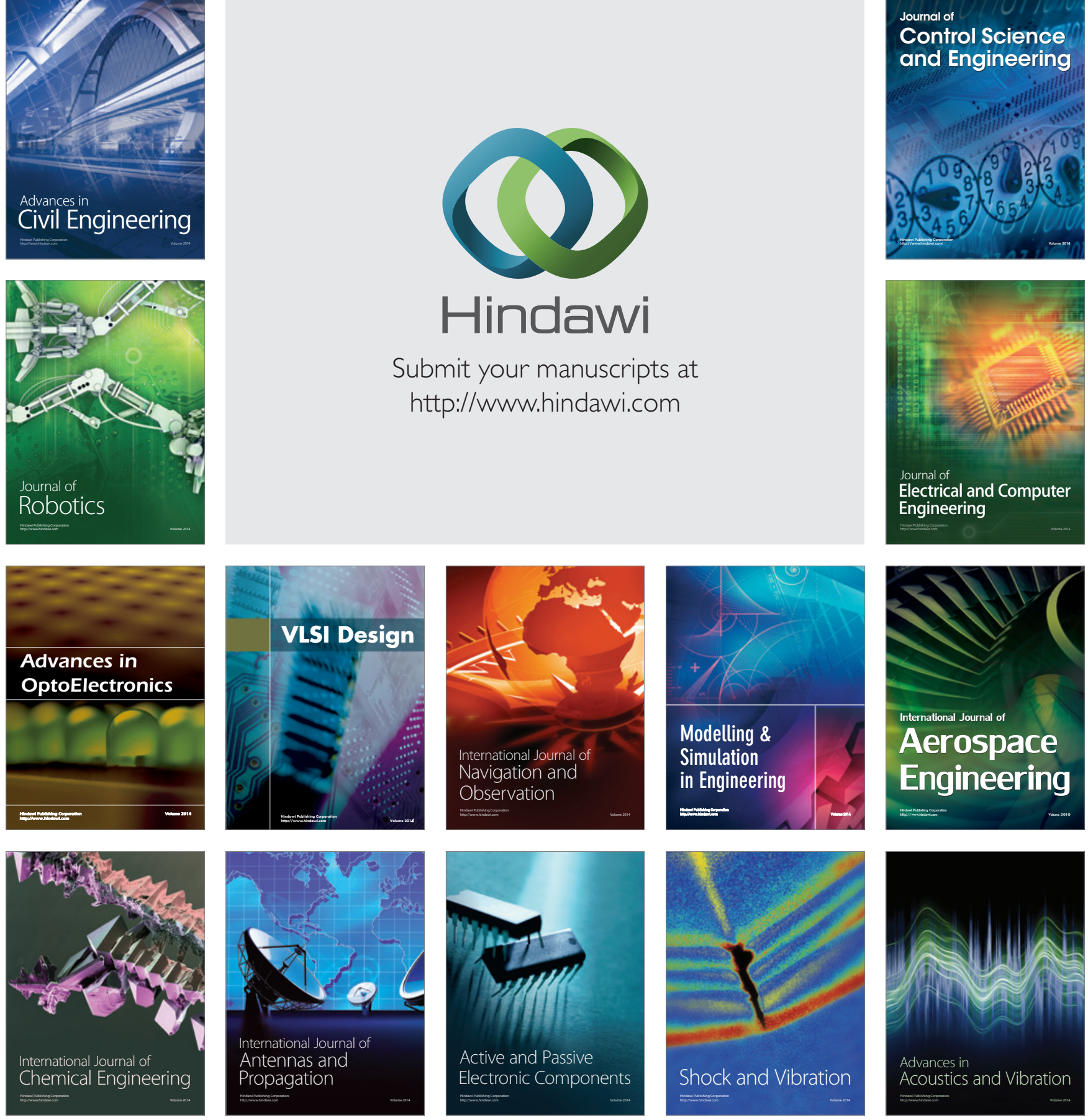